\title{
Vitamin D status and quality of life in people with Irritable Bowel Syndrome
}

\author{
Claire Williams, Elizabeth Williams and Bernard Corde \\ University of Sheffield, Sheffield, United Kingdom
}

\section{Abstract}

Irritable Bowel Syndrome (IBS) is a chronic and debilitating functional disorder of the gastrointestinal tract with serious and detrimental impacts on quality of life. Its aetiology is largely unknown, and the identification of effective management strategies remains far from complete. Research suggests that people with IBS have a high prevalence of vitamin D deficiency, which may impact on symptom severity and quality of life.

A double-blind, placebo-controlled, 2-arm, parallel-design study is underway to investigate the effect of a 12 week, 3,000IU vitamin $\mathrm{D}$ p.d. supplementation on symptom severity and quality of life in participants with a clinical diagnosis of IBS. This report assesses whether relationships exist between vitamin D status, IBS severity and quality of life in the baseline (cross-sectional) data.

Eighty males and females with a clinical diagnosis of IBS were recruited in phase I of the trial in January-April 2018. Mixed-type IBS and constipation-predominant IBS were the more frequent subtypes $(36 \%$ and $33 \%)$, while diarrhoea-predominant had a lower prevalence $(23 \%)$. More females $(n=65)$ than males $(n=15)$ were recruited to the study which reflects the reported greater prevalence of IBS in women. Baseline serum 25(OH)D levels in participants were classified as deficient $23.1 \%(\mathrm{n}=18)(<30 \mathrm{nmol} / \mathrm{L})$, insufficient $37.2 \%(\mathrm{n}=29)(31-49 \mathrm{nmol} / \mathrm{L})$ and sufficient 39.7\% $(\mathrm{n}=31)(>50 \mathrm{nmol} / \mathrm{L})$ respectively. The sample median for serum vitamin $\mathrm{D}$ at baseline was $42.2( \pm 28.3) \mathrm{nmol} / \mathrm{L}$. Quality of Life was significantly associated with two IBS sub-symptoms: pain $(p=0.037, r=0.233)$ and distension severity $(\mathrm{p}=0.009, \mathrm{r}=0.291)$. Neither Quality of Life, nor IBS symptom severity score, correlated with vitamin D status in the sample.

This study found no relationship between baseline vitamin D status and symptom severity or quality of life in this subsample. Two publications report vitamin D intervention improves quality of life in participants with IBS. It is possible that the unusual homogeneity of these studies enabled identification of these relationships.

These initial observations agree with previous reports of widespread vitamin D insufficiency in people with IBS. Analysis of full trial outcomes (July 2019) will determine whether vitamin D supplementation is beneficial for either symptoms severity or quality of life in people with IBS.

\section{Conflict of Interest}

There is no conflict of interest. 